\title{
Spinal cord compression secondary to intrathecal catheter-induced granuloma: a report of four cases
}

\author{
Authors Paul M Arnold, Viraat Harsh, Seth M Oliphant \\ Institution University of Kansas Medical Center, Kansas City, KS, USA
}

\section{ABSTRACT}

Objective: The management of nonmalignant pain by morphine pump implantation has become an effective and increasingly frequent strategy of care. We report a rare complication of intrathecal granuloma formation adjacent to the intrathecal catheter tip resulting in spinal cord compression in four patients undergoing intrathecal treatment for chronic pain.

Methods: Four patients presented with chronic back pain and lower extremity pain and weakness and were treated with morphine pump implantation. Each patient developed a mass at the level of the intrathecal catheter tip resulting in increased back pain and diminished neurological function. Following clinical examination and x-ray workup, the patients underwent surgical resection of the mass and removal of the intrathecal catheter. One patient received conservative saline therapy first, and another patient had granuloma resection first and removal of the intrathecal catheter at a later date. Pathological analysis showed granulation tissue with extensive necrosis and chronic inflammation, with negative culture results. No evidence of neoplasm was found.

Results: Patients showed varying degrees of improvement following removal of the intrathecal mass. Two patients had moderate pain reduction following resection of the granuloma; a third had minimal pain improvement; and a fourth had significant pain improvement but continued lower extremity weakness.

Conclusions: The formation of granulomas caused by intrathecal catheter implantation is a rare but serious complication. Imaging studies including magnetic resonance imaging with contrast and computed tomography with myelogram should be used to follow up a neurological examination consistent with spinal cord compression. Timely surgical intervention may result in marked improvement of symptoms. 


\section{INTRODUCTION}

Initially used in the treatment of cancer pain [1], the implantation of morphine pumps has become an increasingly used treatment for patients with nonmalignant pain. More than 95,000 intrathecal drug delivery devices have been implanted since their inception in the 1980s [2]. The benefits of intrathecal drug therapy for the treatment of nonmalignant pain, most frequently due to "failed back syndrome," have been well documented [3, 4]. A rare but serious complication of this therapy is the development of an inflammatory mass at the catheter tip occurring in less than $3 \%$ of all patients with intrathecal catheters, often resulting in spinal cord compression [2]. The appearance of the developing mass often begins after an extended period of intrathecal morphine therapy, with a sudden increase in pain followed by development of neurological symptoms. We report four patients who underwent intrathecal analgesic treatment, who later presented with symptoms of spinal cord compression and were diagnosed as having developed catheter-tip masses 3.5 to 12 years after intrathecal catheters placement.

\section{CASE REPORT}

\section{Patient 1}

A 51-year-old man presented with a several-month course of increasing chronic low back pain and bilateral lower extremity weakness associated with burning, numbness, and foot pain. His medical history includes two back surgeries with laminectomy and posterior fusion of L4-S1, and was 5 years status after placement of an intrathecal morphine pump for chronic lower back pain. A motor examination of his lower extremities revealed some antalgic weakness bilaterally in both distal lower extremities, especially at dorsi and plantar flexion bilaterally at 4-/5. His knee extension and flexion were full. His right knee jerk was slightly brisk compared with the left.

A magnetic resonance imaging (MRI) of the thoracic and lumbar spine with and without contrast revealed an intradural extramedullary mass at Tll with abnormal hyperintense signal intensity within the spinal cord at the T10-11 levels, suggesting edema or myelomalacia (Fig 1).

The patient underwent T11-12 laminectomy with intradural exploration, resection of the intradural mass, and removal of the intrathecal catheter. Pathological findings showed multiple small fragments of necrotic and fibrous tissue with chronic inflammation and scant hemosiderin consistent with a granuloma. Cultures were negative.

Follow-up neurological examination showed significant improvement in lower extremity and back pain; however, the right lower extremity weakness did not show any improvement. The patient continued oral medication therapy, and the neurological examination was unchanged at 30 months postoperatively.

\section{Patient 2}

A 65-year-old woman presented with mid-to-low back pain radiating down to the thighs, right worse than the left, and progressive weakness and numbness in both lower extremities. She had been administered intrathecal morphine for 12 years for failed back syndrome status after multiple fusions and laminectomies.

A CT myelogram showed an $8 \mathrm{~mm}$ rounded density corresponding to the location of the intrathecal catheter tip at T11-T12 (Fig 2). Physical examination revealed bilateral lower extremity motor strength at $4 / 5$ and diminished lower extremity deep tendon reflexes, while the sensory system was intact. An MRI scan showed a posterior lower thoracic intradural extramedullary mass consistent with granuloma. 
The patient underwent T11 laminectomy with intradural exploration and subsequent removal of intradural mass, which was found to be causing marked spinal cord compression. There were also nerve roots attached to the left lateral aspect of the mass. With the use of microdissectors, the mass was circumferentially freed and removed en block. Pathological findings revealed hyalinized soft tissue with necrosis consistent with granuloma. Cultures were negative. Her pain improved after surgery and she received oral oxycodone for further pain control. Twelve months after follow-up she has no evidence of recurrent granuloma.

Fig 1 T1 sagittal magnetic resonance imaging with contrast shows enhancing lesion at $\mathrm{T} 11$.

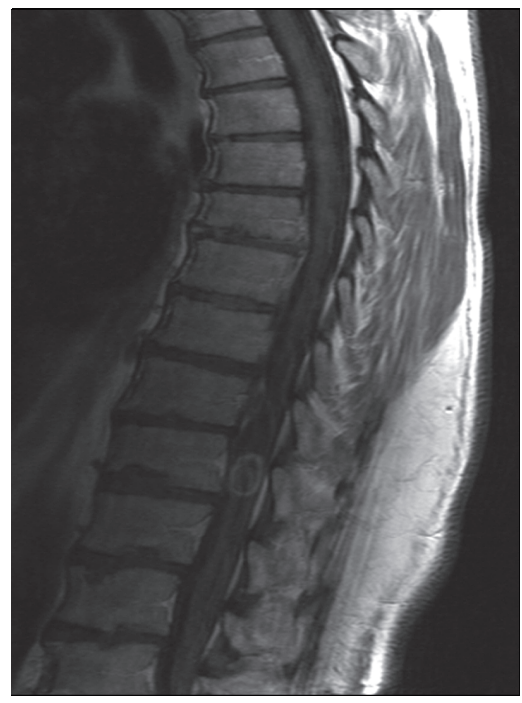

Fig 2 Post-myelogram computed tomography shows granuloma at $\mathrm{T} 11$.

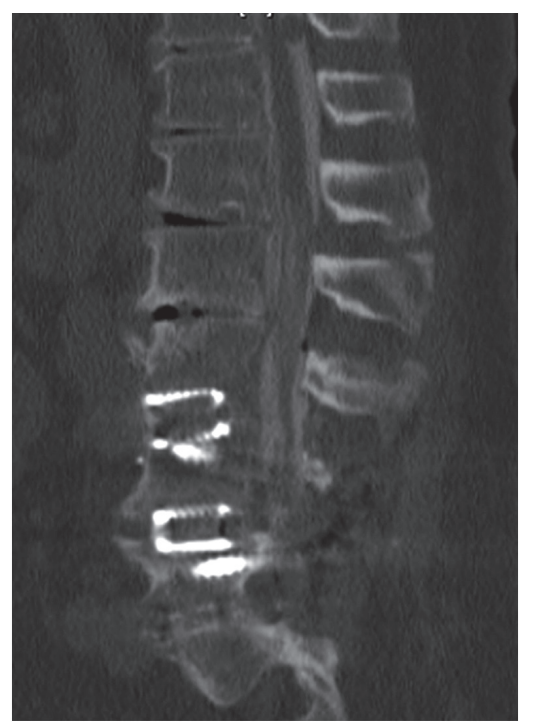

\section{DISCUSSION}

The intrathecal effect of morphine in the treatment of chronic pain is through spinal and supraspinal receptors, without significantly influencing motor, sensory, and sympathetic reflexes [4]. It is most often used in the nonmalignant patient for chronic lumbosacral pain due to "failed back syndrome" [5]. Animal studies have shown that with chronic intrathecal infusion of the maximum tolerated doses of morphine, hydromorphone, L-methadone, and naloxone, there is $100 \%$ intradural granuloma formation [6]. In humans the duration of therapy before granuloma diagnosis has been reported to be 0.5 to 72 months [7]. After 2 years of therapy, the incidence of symptomatic intrathecal catheter-tip granuloma formation is reported to be only $0.4 \%$; after 6 years, this incidence increases to only $1.16 \%[8,9]$. Three of our four cases were within this time frame, while one case was diagnosed with intrathecal granuloma after 12 years of intrathecal catheter placement.

The other two cases not previously discussed presented with symptoms of spinal cord compression and were diagnosed with intrathecal granuloma 3.5 years and 5 years, respectively, after intrathecal catheter placement. The former was treated with the granuloma being removed first and the intrathecal catheter removed at a later date; the latter was initially treated conservatively with pump refills replaced with saline. None of these management methods were found to be effective and both patients had to ultimately undergo removal of the intrathecal catheter and resection of the granuloma. The first patient had minimal pain improvement. The second patient was discharged in good condition; he remains in continued pain 2 years after granuloma resection.

To date, less than 60 cases of inflammatory catheter-tip masses have been reported following chronic infusion of opiates [4, 10-28]. Four of these showed the development of a granuloma that included findings of positive cultures, with one occurring 11 years after discontinuation of the opiate therapy; infection is unlikely as the cause for the other three $[11-13,17]$. Three cases have also been published where the patient developed inflammatory cathetertip masses with baclofen as the sole agent in a long-term intrathecal catheter [15, 29]. Hydromorphone has also been found to cause intrathecal granuloma formation [23]. In animal model studies, baclofen alone had not been shown to induce granuloma formation with long-term intrathecal delivery [29]. 
The etiology for the development of inflammatory masses caused by intrathecal morphine is unclear. Proposed mechanisms include the action of morphine as a mitogen, activating a protein kinase cascade and activating lymphocyte activity. Another is the effect of opioids on endothelial cells, granulocytes, and monocytes to release nitric oxide, which may lead to monocyte migration, or finally that morphine enhances cytokine formation leading to the inflammatory response [30]. The catheter tip being positioned in the thoracic spinal cord, which is the longest area of low cerebrospinal fluid velocity, in conjunction with use of high concentration of drugs is also considered a contributing factor to inciting local inflammation. The resulting fibrosis further decreases cerebrospinal fluidflow velocity and turns the situation into a vicious cycle, amplifying the drug concentration in that area [2].

The treatment of intrathecal inflammatory masses in patients with chronic pain includes surgical and nonsurgical methods. Nonsurgical methods, as conservative saline therapy, have not been found to be very beneficial; while surgical resection of the mass and removal of the intrathecal catheter provided the most satisfactory results for our four case-patients. The nonsurgical treatment by discontinuation of opiates has been beneficial in some patients; however, our patient who was treated with this method continued to have a progression of symptoms, and surgical management was required [30]. Surgery may be the optimal treatment for symptomatic granulomas compressing the spinal cord.

\section{Fig 3 Patient sampling and selection.}

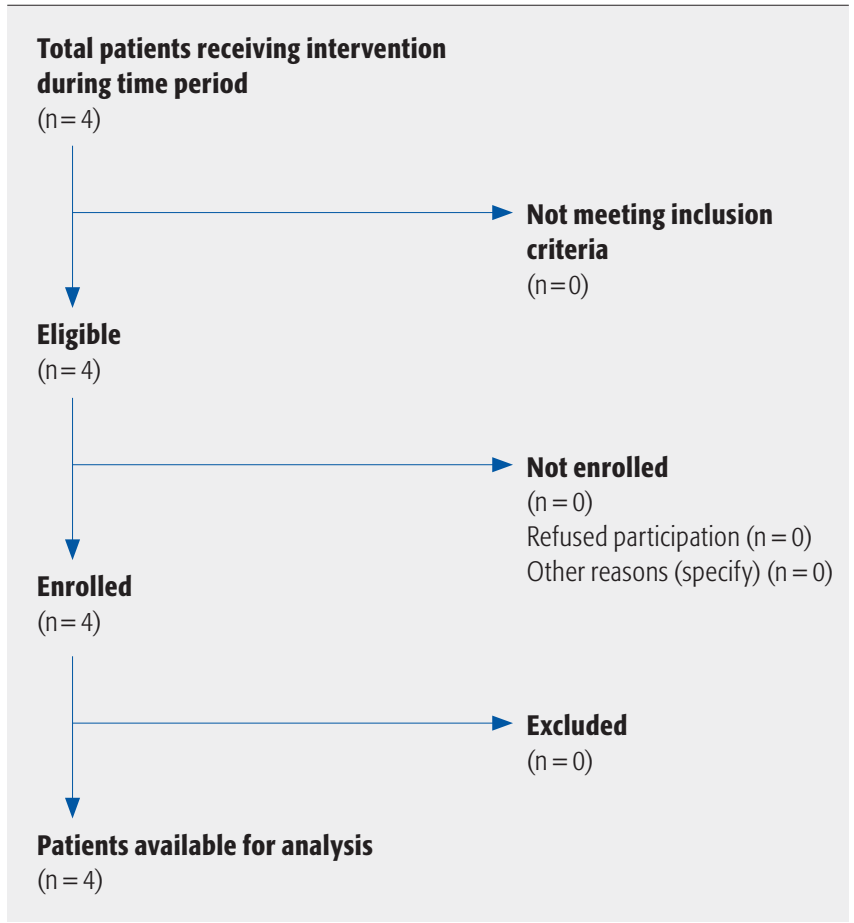

\section{SUMMARY AND CONCLUSION}

The formation of granulomas induced by intrathecal catheter implantation is a rare but serious complication. Imaging studies including MRI with contrast and CT myelogram should be used to follow up a neurological examination consistent with spinal cord compression. Timely surgical intervention may result in marked improvement of symptoms.

\section{ACKNOWLEDGMENT}

We thank Karen K Anderson for her assistance in manuscript preparation. 


\section{REFERENCES}

1. Magill ST, Wang $\mathbf{P}$, Eller JL, et al (2008) Differentiating intrathecal catheter tip granulomas from normal magnetic resonance image distortion caused by metallic catheter tips. Neurosurgery; 62(1):242-248.

2. Medel R, Pouratian N, Elias WJ (2010) Catheter-tip mass mimicking a spinal epidural hematoma: case report. J Neurosurg Spine; 12(1):66-71.

3. Schuchard M, Krames ES, Lanning RM (1998) Intraspinal analgesia for nonmalignant pain: a retrospective analysis for efficacy, safety, and feasibility in 50 patients. Neuromodulation; 1:46-56.

4. Winkelmüller M, Winkelmüller W (1996) Long-term effects of continuous intrathecal opioid treatment in chronic pain of nonmalignant etiology. J Neurosurg; 85(3):458-467.

5. Miele VJ, Price KO, Bloomfield S, et al (2006) A review of intrathecal morphine therapy related granulomas. Eur J Pain; 10(3):251-261.

6. Allen JW, Horais KA, Tozier NA, et al (2006) Opiate pharmacology of intrathecal granulomas. Anesthesiology; 105(3):590-598.

7. Deer TR (2004) A prospective analysis of intrathecal granuloma in chronic pain patients: a review of the literature and report of a surveillance study. Pain Physician; 7(2):225-228.

8. Yaksh T, Hassenbusch S, Burchiel $\mathrm{K}$, et al (2002) Inflammatory masses associated with intrathecal drug infusion: a review of preclinical evidence and human data. Pain Med; 3:300-312.

9. Ruan X (2007) Drug-related side effects of long-term intrathecal morphine therapy. Pain Physician; 10(2):357-366.

10. Aldrete JA, Vascello LA, Ghaly R, et al (1994) Paraplegia in a patient with an intrathecal catheter and a spinal cord stimulator. Anesthesiology; 81(6):1542-1545.

11. Bejjani GK, Karim NO, Tzortzidis F (1997) Intrathecal granuloma after implantation of a morphine pump: case report and review of the literature. Surg Neurol; 48(3):288-291.

12. Blount JP, Remley KB, Yue SK, et al (1996) Intrathecal granuloma complicating chronic spinal infusion of morphine: report of three cases. J Neurosurg; 84(2):272-276.

13. Cabbell KL, Taren JA, Sagher $O$ (1998) Spinal cord compression by catheter granulomas in high-dose intrathecal morphine therapy: case report. Neurosurgery; 42(5):1176-1180.

14. Coffey RJ, Burchiel K (2002) Inflammatory mass lesions associated with intrathecal drug infusion catheters: report and observations on 41 patients. Neurosurgery; 50(1):78-86.

15. Deer TR, Raso LJ, Garten TG (2007) Inflammatory mass of an intrathecal catheter in patients receiving baclofen as a sole agent: a report of two cases and a review of the identification and treatment of the complication. Pain Med; 8(3):259-262.

16. Langsam A (1999) Spinal cord compression by catheter granulomas in high-dose intrathecal morphine therapy: case report. Neurosurgery; 44(3):689-691.

17. Lehmberg J, Scheiwe C, Spreer J, et al (2006) Late bacterial granuloma at an intrathecal drug delivery catheter. Acta Neurochir (Wien); 148(8):899-901.

18. McMillan MR, Doud T, Nugent W (2003) Catheter-associated masses in patients receiving intrathecal analgesic therapy. Anesth Analg; 96(1):186-190.

19. Meta G, Ghaleb A, Gentry WB, et al (2006) Intrathecal therapy-associated masses. Anesth Analg; 103(1):260-261.

20. Miele VJ, Price K, Bloomfield S, et al (2006) Intrathecal morphine therapy related granulomas: two case reports. $W$ V Med J; 102(5):16-18.

21. North RB, Cutchis PN, Epstein JA, et al (1991) Spinal cord compression complicating subarachnoid infusion of morphine: case report and laboratory experience. Neurosurgery; 29(5):778-784.

22. Peng P, Massicotte EM (2004) Spinal cord compression from intrathecal catheter-tip inflammatory mass: case report and a review of etiology. Reg Anesth Pain Med; 29(3):237-242.

23. Ramsey CN, Owen RD, Witt Wo, et al (2008) Intrathecal granuloma in a patient receiving high dose hydromorphone. Pain Physician; 11(3):369-373.

24. Rodan BA, Cohen FL, Bean WJ, et al (1985) Fibrous mass complicating epidural morphine infusion. Neurosurgery; 16(1):68-70.

25. Schuchard $M$, Lanning $R$, North $R$, et al (1998) Neurologic sequelae of intraspinal drug delivery systems: results of a survey of American implanters of implantable drug delivery systems. Neuromodulation; 1:137-148.

26. Shields DC, Palma C, Khoo LT, et al (2005) Extramedullary intrathecal catheter granuloma adherent to the conus medullaris presenting as cauda equina syndrome. Anesthesiology; 102(5):1059-1061.

27. De Andrés J, Palmisani S, Villanueva Pérez VL, et al (2010) Can an intrathecal, cathetertip-associated inflammatory mass reoccur? Clin J Pain; 26(7):631-634.

28. Jhas S, Tuli S (2008) Intrathecal catheter-tip inflammatory masses: an intraparenchymal granuloma. J Neurosurg Spine; 9(2):196-199.

29. Murphy PM, Skouvaklis DE, Amadeo RJ, et al (2006) Intrathecal catheter granuloma associated with isolated baclofen infusion. Anesth Analg; 102(3):848-852.

30. Hassenbusch S, Burchiel K, Coffey RJ, et al (2002) Management of intrathecal catheter-tip inflammatory masses: a consensus statement. Pain Med; 3(4):313-323. 


\section{COMMENTARY: \\ "SPINAL CORD COMPRESSION SECONDARY TO INTRATHECAL CATHETER-INDUCED GRANULOMA: A REPORT OF FOUR CASES “}

\author{
Author Jean-Paul Wolinsky \\ Institution Department of Neurosurgery, The Johns \\ Hopkins Hospital, Baltimore, MD, USA
}

The authors report on four cases of symptomatic granuloma formation at the catheter tip of intradural morphine pumps. They discuss the clinical presentation of these patients, the surgical treatment, and the outcomes. In addition, they present a review of the literature with regard to the pathology of this entity and the treatment of this problem.

The paper is presented and formatted as an original research submission and prognostic study. It appears to be a retrospective case series of patients with symptomatic granulomas in the location of the catheter tip of an intradural morphine pump. There is no treatment algorithm that is presented or tested. Figure 3 attempts to present the series as a prospective trial, but there is no evidence that this was the case. In addition, this figure is not referenced and appears to be added at the end of the paper without any significant purpose. Although the authors present an interesting series of patients, their observations add little to the literature to further our understanding of the pathology and treatment of this entity.

The authors present a review of the literature with regard to intrathecal morphine pumps and intradural granuloma formation. The review is not designed as a meta-analysis to determine the best treatment options, but as an update of the current understanding of this entity. They have highlighted and referenced excellent articles with regard to this pathology, and this review provides a worthwhile update. Specifically, three references that merit mentioning are cited here:

Hassenbusch S, Burchiel K, Coffey RJ, et al (2002) Management of intrathecal catheter-tip inflammatory masses: a consensus statement. Pain Med; 3(4):313-23.

Coffey RJ, Burchiel K (2002) Inflammatory mass lesions associated with intrathecal drug infusion catheters: report and observations on 41 patients. Neurosurgery; 50(1):78-86.

Yaksh T, Hassenbusch S, Burchiel K, et al (2002) Inflammatory masses associated with intrathecal drug infusion: a review of preclinical evidence and human data. Pain Med; 3:300-312.

\section{EDITORIAL STAFF PERSPECTIVE}

Intrathecal catheters, pumps, and electrodes are more commonly deployed than ever before for various reasons revolving mainly around pain relief. The foundational evidence for their application, such as superiority of pain relief or greater safety remains less than robust and has recently been called into question by some governmental review organizations (www.hta. hca.wa.gov:80/pumps.html).

Clearly, the potential for more long-term serious but unforeseeable complications with application of newer technologies should be almost anticipated. Case reports can serve a valuable 'canary in a coal mine' function in reporting adverse events. Hopefully increased awareness of complications will lead to more systematic review of their rate of occurrence and understanding of causation as well as inclusion into patient disclosures rather than prompt overzealous regulatory kneejerk reactions. 